\title{
A PRESENTATION FOR HILDEN'S SUBGROUP OF THE BRAID GROUP
}

\author{
STEPHEN TAWN
}

\begin{abstract}
Consider the unit ball, $B=D \times[0,1]$, containing $n$ unknotted $\operatorname{arcs} a_{1}, \ldots, a_{n}$ such that the boundary of each $a_{i}$ lies in $D \times\{0\}$. We give a finite presentation for the mapping class group of $B$ fixing the arcs $\left\{a_{1}, \ldots, a_{n}\right\}$ setwise and fixing $D \times\{1\}$ pointwise. This presentation is calculated using the action of this group on a simplyconnected complex.
\end{abstract}

\section{Introduction}

Let $H^{3}$ denote the closed upper half-space of $\mathbb{R}^{3}$, let $a_{1}, a_{2}, \ldots, a_{n} \subset H^{3}$ be $n$ pairwise disjoint properly embedded unknotted arcs and let $a_{*}=a_{1} \cup a_{2} \cup \cdots \cup a_{n}$. Viewing the braid group as the mapping class group of the punctured disc, if this disc is included in $\partial H^{3}$ with $\partial a_{*}$ as the punctures, one can define Hilden's group, $\mathbf{H}_{2 n}$, to be the subgroup of $\mathbf{B}_{2 n}$ consisting of all mapping classes that can be extended to $H^{3} \backslash a_{*}$. Or equivalently, $\mathbf{H}_{2 n}$ is the stabiliser of $a_{*}$ under the action of $\mathbf{B}_{2 n}$ on $0,2 n$-tangles.

Hilden[5] found generators for a similar subgroup of the braid group of a sphere. For any given braid $b$ multiplying on either the left or the right by elements of $\mathbf{H}_{2 n}$ preserves the plat closure, ie plat closure is constant on each double coset. Birman[1] showed that if two braids have the same plat closure then they can be related by a sequence of these double coset moves and stabilisation moves that changes the braid index by 2 .

We calculate a presentation for $\mathbf{H}_{2 n}$ using the action of this group on a cellular complex. Hatcher-Thurston[4], Wajnryb[7, 8, 9], Laudenbach[6], etc used the same method to calculate presentations for mapping class groups. We start in Section 2 by outlining this method. A similar but more general method is given by Brown [3]. Brendle-Hatcher[2] have calculated a presentation for $\mathbf{H}_{2 n}$ using a different method.

In Section 3 we define a simply-connected complex $\overline{\mathbf{X}}_{n}$. In Section 4 we remove some of the edges and faces of this complex resulting in a new complex which remains simply-connected but gives a simpler presentation. This presentation is calculated in Section 5 and then used to calculate a presentation with generators similar to those found by Hilden.

\section{The method}

Suppose that $X$ is a connected simply-connected cellular 2-complex such that each attaching map is injective and that each cell is uniquely determined by its boundary. Suppose that $G$ is a group acting cellularly on the right of $X$, and that this action

Received by the editors October 8, 2007 
is transitive on the vertex set $X^{0}$. Pick a vertex $v_{0} \in X^{0}$ as a basepoint and let $H$ denote its stabiliser in $G$, ie $H=\left\{g \in G \mid v_{0} \cdot g=v_{0}\right\}$. Suppose that $H$ has a presentation with generating set $S_{0}$ and relations $R_{0}$, ie $H=\left\langle S_{0} \mid R_{0}\right\rangle$.

Given vertices $u, v \in X^{0}$ such that $\{u, v\}$ is the boundary of an edge of $X$ we will write $(u, v)$ for this (oriented) edge. Given a sequence $v_{1}, v_{2}, \ldots, v_{k}$ of vertices such that either $v_{i}=v_{i+1}$ or $\left(v_{i}, v_{i+1}\right)$ forms an edge we will write $\left(v_{1}, v_{2}, \ldots, v_{k}\right)$ for the path traversing these edges. Whenever $v_{i}=v_{i+1}$ we shall say that $v_{i}$ is a stationary point.

Let $E$ denote the set of all oriented edges starting at $v_{0}$, so $H$ acts on $E$. Suppose that $\left\{e_{\lambda}\right\}_{\lambda \in \Lambda}$ is a set of representatives for the $H$-orbits of the edges in $E$, ie $E=$ $\bigcup_{\lambda \in \Lambda} H e_{\lambda}$ and $H e_{\lambda}=H e_{\lambda^{\prime}}$ only if $\lambda=\lambda^{\prime}$. Since the action of $G$ is transitive on $X^{0}$ we can find $r_{\lambda} \in G$ such that $e_{\lambda}=\left(v_{0}, v_{0} \cdot r_{\lambda}\right)$. Let $S_{1}=\left\{r_{\lambda}\right\}_{\lambda \in \Lambda}$.

The edges $\left\{e_{\lambda}\right\}_{\lambda \in \Lambda}$ also form a set of representatives for the edge orbits of the $G$-action on $X$. To see this suppose that two of these edges lie in the same $G$-orbit, ie $\left(v_{0}, v\right)=\left(v_{0}, u\right) \cdot g$. Then we have that $v_{0}=v_{0} \cdot g$ therefore $g \in H$.

Suppose that $\left\{f_{\mu}\right\}_{\mu \in M}$ is a set of representatives for the $G$-orbits of the faces of $X$. Since the action is transitive on $X^{0}$, we may assume that the boundary of each face $f_{\mu}$ contains the vertex $v_{0}$.

Definition 2.1. An h-product of length $k$ is a word of the form

$$
h_{k+1} r_{\lambda_{k}} h_{k} r_{\lambda_{k-1}} h_{k-1} \cdots r_{\lambda_{1}} h_{1}
$$

where each $\lambda_{i} \in \Lambda$ and each of the $h_{i}$ are words in $H$. To each h-product we can associate an edge path $P=\left(v_{0}, v_{1}, \ldots, v_{k}\right)$ in $X$ starting at $v_{0}$ then visiting the vertices $v_{1}=v_{0} \cdot r_{\lambda_{1}} h_{1}, v_{2}=v_{0} \cdot r_{\lambda_{2}} h_{2} r_{\lambda_{1}} h_{1}$, etc. This means that the edge $\left(v_{i-1}, v_{i}\right)$ is in the orbit of $\left(v_{0}, v_{0} \cdot r_{\lambda_{i}}\right)$. Given any edge path starting at $v_{0}$ we can choose an h-product to represent it.

We can now choose the following three sets of relations.

$R_{1}$ : For each edge orbit representative $e_{\lambda}$ pick a generating set $T$ for the stabiliser of this edge, ie $\langle T\rangle=\operatorname{Stab}_{G}\left(v_{0}\right) \cap \operatorname{Stab}_{G}\left(v_{0} \cdot r_{\lambda}\right)$. For each $t \in T$ we have the relation $r_{\lambda} t r_{\lambda}^{-1}=h$ for some word $h \in H$.

$R_{2}$ : For each $e_{\lambda}$ we have a relation $r_{\lambda^{\prime}} h r_{\lambda}=h^{\prime}$ where the LHS is a choice of h-product for the path $\left(v_{0}, v_{0} \cdot r_{\lambda}, v_{0}\right)$ and $h^{\prime}$ is some word in $H$.

$R_{3}$ : For each face orbit representative $f_{\mu}$ with boundary $\left(v_{0}, v_{1}, \ldots, v_{k-1}, v_{0}\right)$ choose an h-product representing this path and a word $h \in H$ such that $r_{\lambda_{k}} h_{k} \cdots r_{\lambda_{1}} h_{1}=h$.

Theorem 2.2. The group $G$ has the following presentation.

$$
G=\left\langle S_{0} \cup S_{1} \mid R_{0} \cup R_{1} \cup R_{2} \cup R_{3}\right\rangle
$$

Corollary 2.3. Suppose that $H$ is finitely presented, that the number of edge and face orbits is finite and that each edge stabiliser is finitely generated. Then $G$ has a finite presentation.

We prove Theorem 2.2 in several steps.

Claim 1. The set $S_{0} \cup S_{1}$ generates $G$. 
Proof. Given any $g \in G$, let $v=v_{0} \cdot g$. Now as $X$ is connected there is an edge path connecting $v_{0}$ to $v$. Choose an h-product $g_{1}=h_{k+1} r_{\lambda_{k}} h_{k} \cdots r_{\lambda_{1}} h_{1}$ representing this path. Then $v_{0} \cdot g g_{1}^{-1}=v_{0}$ so $g=h g_{1}$ for some $h \in H$.

Claim 2. If two h-products, $p_{1}$ and $p_{2}$, give rise to the same path and are equal in $G$ then they are equivalent modulo $R_{0} \cup R_{1}$.

Proof. Because $p_{1}$ and $p_{2}$ represent the same path they must have equal length. Suppose that $p_{1}=h_{k+1} r_{\lambda_{k}} h_{k} \cdots r_{\lambda_{1}} h_{1}$ and $p_{2}=f_{k+1} r_{\lambda_{k}^{\prime}} f_{k} \cdots r_{\lambda_{1}^{\prime}} f_{1}$. Clearly, if the two h-products are of length 0 then they are both words in $H$ and so are equivalent modulo $R_{0}$. Now suppose that $k \neq 0$. The fact that $p_{1}$ and $p_{2}$ represent the same path means that

$$
\left(v_{0}, v_{0} \cdot r_{\lambda_{1}} h_{1}, v_{0} \cdot r_{\lambda_{2}} h_{2} r_{\lambda_{1}} h_{1}, \ldots\right)=\left(v_{0}, v_{0} \cdot r_{\lambda_{1}^{\prime}} f_{1}, v_{0} \cdot r_{\lambda_{2}^{\prime}} f_{2} r_{\lambda_{1}^{\prime}} f_{1}, \ldots\right),
$$

therefore

$$
\left(v_{0}, v_{0} \cdot r_{\lambda_{1}}\right)=\left(v_{0}, v_{0} \cdot r_{\lambda_{1}^{\prime}}\right) \cdot f_{1} h_{1}^{-1} .
$$

So $\lambda_{1}=\lambda_{1}^{\prime}$ and $f_{1} h_{1}^{-1}$ is in the stabiliser of the edge $e_{\lambda_{1}}$. Hence, for some word $f_{2}^{\prime}$ in $H$

$$
f_{k+1} r_{\lambda_{k}^{\prime}} f_{k} \cdots r_{\lambda_{2}^{\prime}} f_{2} r_{\lambda_{1}^{\prime}} f_{1} h_{1}^{-1} h_{1}=f_{k+1} r_{\lambda_{k}^{\prime}} f_{k} \cdots r_{\lambda_{2}^{\prime}} f_{2}^{\prime} r_{\lambda_{1}} h_{1}
$$

modulo $R_{1}$. By induction the two shorter h-products $h_{k+1} r_{\lambda_{k}} h_{k} \cdots r_{\lambda_{2}} h_{2}$ and $f_{k+1} r_{\lambda_{k}^{\prime}} f_{k} \cdots r_{\lambda_{2}^{\prime}} f_{2}^{\prime}$ are equivalent modulo $R_{0} \cup R_{1}$, and so $p_{1}=p_{2}$ modulo $R_{0} \cup R_{1}$.

Claim 3. Suppose that two h-products represent the same element of $G$ and induce edge paths that are equivalent modulo backtracking. Then they are equivalent modulo $R_{0} \cup R_{1} \cup R_{2}$.

Proof. It is enough to show that any h-product is equivalent to an h-product that represents a path without any backtracking. Furthermore, if we proceed by induction on the length of the h-product, it is enough to show that any h-product whose associated path has backtracking at the end is equivalent to a shorter h-product.

Suppose that $g=h_{k+3} r_{\lambda_{k+2}} h_{k+2} r_{\lambda_{k+1}} h_{k+1} g_{k}$ is such an h-product, ie

$$
\begin{aligned}
v_{k} & =v_{0} \cdot g_{k} \\
v_{k+1} & =v_{0} \cdot r_{\lambda_{k+1}} h_{k+1} g_{k} \\
v_{k+2}=v_{k} & =v_{0} \cdot r_{\lambda_{k+2}} h_{k+2} r_{\lambda_{k+1}} h_{k+1} g_{k}
\end{aligned}
$$

and $g_{k}$ is a shorter h-product. So, multiplying by $g_{k}^{-1} h_{k+1}^{-1}$, we find that $r_{\lambda_{k+2}} h_{k+2} r_{\lambda_{k+1}}$ is an h-product with associated path $\left(v_{0}, v_{0} \cdot r_{\lambda_{k+1}}, v_{0}\right)$. Suppose that $r_{\lambda^{\prime}} h r_{\lambda}=h^{\prime}$ is the $R_{2}$ relation corresponding to this path. Then $\lambda=\lambda_{k+1}$ and $v_{0} \cdot r_{\lambda^{\prime}} h=v_{0} \cdot r_{\lambda_{k+2}} h_{k+2}$. So $\lambda^{\prime}=\lambda_{k+2}$ and $h_{k+2} h^{-1}$ is in the stabiliser of the edge $e_{\lambda_{k+1}}$. Therefore there exists a word $f$ in $H$ such that

$$
h_{k+3} r_{\lambda_{k+2}} h_{k+2} r_{\lambda_{k+1}} h_{k+1} g_{k}=h_{k+3} f r_{\lambda^{\prime}} h r_{\lambda} h_{k+1} g_{k}
$$

modulo $R_{1}$. Hence modulo $R_{2}$ this is equal to $h_{k+3} f h^{\prime} h_{k+1} g_{k}$, a shorter h-product.

Claim 4. Any h-product equal to the identity in $G$ is equivalent to the identity modulo $R_{0} \cup R_{1} \cup R_{2} \cup R_{3}$. 
Proof. Given any h-product $g_{k}$ equal to the identity in $G$ its associated edge path must be a loop. Since $X$ is simply-connected this loop is the boundary of a union of faces of $X$. So choose one of these faces $f$ touching the loop at a vertex $v$ then modulo $R_{0} \cup R_{1} \cup R_{2}$ we can add backtracking starting at $v$ going around the boundary of $f$. Modulo $R_{3}$ we can remove one pass round $\partial f$. This leaves a new loop that can be spanned by one less face, which, by induction on the minimum number of faces needed to span a loop, is equivalent to the identity.

Proof of Theorem 2.2. Given any word in the generators, $S_{0} \cup S_{1}$, that is equal to the identity in $G$ then modulo $R_{2}$ it is equivalent to an h-product and so by Claim 4 is equivalent to the identity modulo $R_{0} \cup R_{1} \cup R_{2} \cup R_{3}$.

\section{The complex $\overline{\mathbf{X}}_{n}$}

An embedded disc $D \subseteq H^{3}$ is said to cut out $a_{i}$ if the interior of $D$ is disjoint from $a_{*}$, the arc $a_{i}$ is contained in the boundary of $D$ and the boundary of $D$ lies in $a_{i} \cup \partial H^{3}$, ie $a_{i} \subset \partial D$ and $\partial D \subset a_{i} \cup \partial H^{3}$. A cut system for $a_{*}$ is the isotopy class of $n$ pairwise disjoint discs $\left\langle D_{1}, D_{2}, \ldots D_{n}\right\rangle$ where each $D_{i}$ cuts out the arc $a_{i}$. Say that two cut systems $\left\langle D_{1}, D_{2}, \ldots, D_{n}\right\rangle$ and $\left\langle E_{1}, E_{2}, \ldots, E_{n}\right\rangle$ differ by a simple $i$-move if $D_{i} \cap E_{i}=a_{i}$ and $D_{j}=E_{j}$ for all $j \neq i$. If this is the case we will suppress the non-changing discs and write $\left\langle D_{i}\right\rangle \rightarrow\left\langle E_{i}\right\rangle$.

Definition 3.1. Define the cut system complex $\overline{\mathbf{X}}_{n}$ as follows. The set of all cut systems for $a_{*}$ forms the vertex set $\overline{\mathbf{X}}_{n}^{0}$. Two vertices are connected by a single edge iff they differ by a simple move. Finally, glue faces into every loop of the following form, giving triangular and rectangular faces.
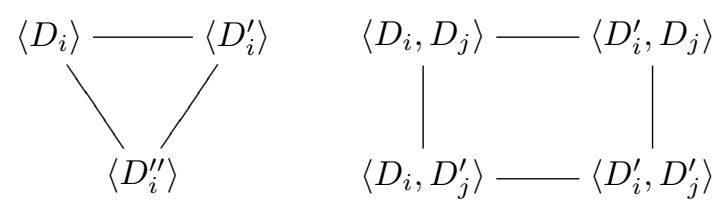

Define the basepoint to be $v_{0}=\left\langle d_{1}, d_{2}, \ldots, d_{n}\right\rangle$ where the $d_{i}$ are vertical discs below the $a_{i}$, see Figure 1. Sometimes it is convenient to think of the $a_{i}$ and $d_{i}$ rotated by

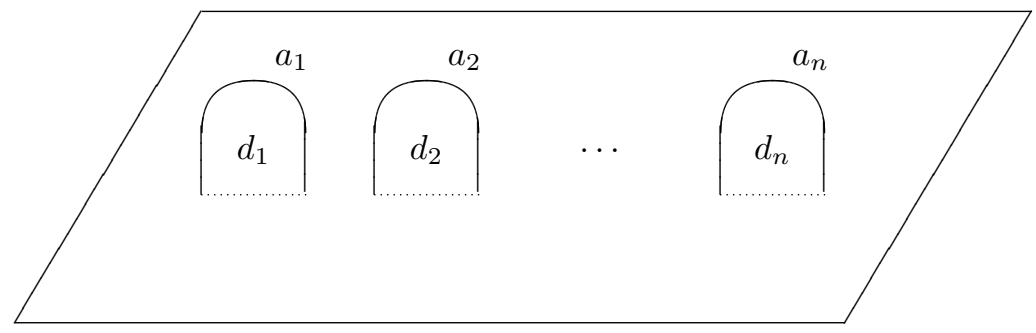

Figure 1. The $\operatorname{arcs} a_{i}$ and the discs $d_{i}$

a quarter turn. 
Before we prove that this complex is simply connected we need the following lemma about substituting one disc for another.

Suppose that $v=\left\langle D_{1}, D_{2}, \ldots D_{n}\right\rangle$ is a vertex of $\overline{\mathbf{X}}_{n}$ and that $D$ and $D^{*}$ are two discs cutting out the arc $a_{i}$. We will say that the tuple $\left(v, D, D^{*}\right)$ forms a valid substitution if either $D \neq D_{i}$ for any $i$, or if there exists some $i$ such that $D=D_{i}$ and that for all $j \neq i$ we have that $D_{j} \cap D^{*}=\emptyset$. In other words if $D$ is in $v$ then $\left(v, D, D^{*}\right)$ forms a valid substitution if there exists an edge $\left\langle D=D_{i}\right\rangle-\left\langle D^{*}\right\rangle$. If $\left(v, D, D^{*}\right)$ forms a valid substitution then we can replace $D$ with $D^{*}$ to get a vertex $v^{*}$, ie

$$
v^{*}= \begin{cases}v & \text { if } D_{i} \neq D, \\ \left\langle D^{*}\right\rangle & \text { if } D_{i}=D .\end{cases}
$$

Similarly, for any edge path $P$ with a choice of discs representing each vertex, we say $\left(P, D, D^{*}\right)$ forms a valid substitution if for each vertex $v$ of $P$ the tuple $\left(v, D, D^{*}\right)$ forms a valid substitution and for each edge $\left(v_{i}, v_{i+1}\right)$ of $P$ there is an edge $\left(v_{i}^{*}, v_{i+1}^{*}\right)$. If $\left(P, D, D^{*}\right)$ forms a valid substitution then we can replace each occurrence of $D$ with $D^{*}$, ie replace each vertex $v$ with $v^{*}$, giving a new path $P^{*}$.

Lemma 3.2. If $\left(P, D, D^{*}\right)$ forms a valid substitution, where $P=\left(v_{1}, \ldots, v_{k}\right)$, then $P^{*}$ is a path and the loop

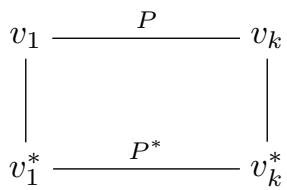

is homotopic to a point. Moreover, if $P$ is a loop then so is $P^{*}$ and they are homotopic as loops.

Proof. Clearly we may assume that $D$ and $D^{*}$ are not isotopic, otherwise $P=P^{*}$. Suppose that $D$ and $D^{*}$ cut out the $\operatorname{arc} a_{i}$. For each vertex $v$ of $P$ we have that either $v=v^{*}$ or $\left(v, v^{*}\right)$ is an edge of $\overline{\mathbf{X}}_{n}$.

For each edge $(u, v)$ in $P$, where $u=\left\langle D_{j}\right\rangle$ and $v=\left\langle D_{j}^{\prime}\right\rangle$, we have the following possibilities. If $D$ is not in $u$ nor in $v$ then $(u, v)=\left(u^{*}, v^{*}\right)$. Otherwise we have two cases depending on whether $i=j$ or not.

If $i=j$ then only one of either $u$ or $v$ contains $D$. Suppose that $D \in u$, ie $D_{j}=D$. If $D^{*}=D_{j}^{\prime}$ then $u^{*}=v^{*}=v$ and $(u, v)$ is homotopic to $\left(u^{*}, v^{*}\right)$ in $\overline{\mathbf{X}}_{n}^{1}$. Otherwise, if $D^{*} \neq D_{j}$, we have the following face of $\overline{\mathbf{X}}_{n}$.

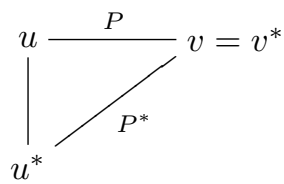

If $i \neq j$ the we have the following face of $\overline{\mathbf{X}}_{n}$.

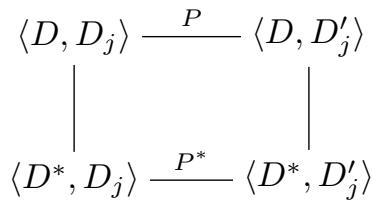


In either case there is a homotopy from $(u, v)$ to $\left(u^{*}, v^{*}\right)$ that agrees with the homotopies between the vertices of $P$ and $P^{*}$. Therefore $P$ is homotopic to $P^{*}$.

Theorem 3.3. The complex $\overline{\mathbf{X}}_{n}$ is connected and simply connected.

Proof. It suffices to show that any loop is homotopic to the constant loop at $v_{0}$. Given a loop in $\overline{\mathbf{X}}_{n}$ it is homotopic to an edge path $P$. Now choose discs to represent each vertex of $P$. We shall write $D \in P$ if $D$ is one of the discs chosen as a representative of some vertex of $P$.

Claim. The path $P$ is homotopic to a path whose vertices admit representative discs which intersect the discs $d_{1}, d_{2}, \ldots, d_{n}$ only in the arcs $a_{1}, a_{2}, \ldots, a_{n}$.

Assuming that the intersection of the discs $D \in P$ with $d_{1} \cup d_{2} \cup \ldots \cup d_{n}$ isn't only $a_{1}, a_{2}, \ldots, a_{n}$ we can carry out the following procedure.

For some $i$ the union of the discs in $P$ intersects $d_{i}$ in a non-empty collection of arcs. Pick an arc $\alpha$ of this intersection that is lowest in the sense that it doesn't separate the entirety of any other arc from $\partial H^{3} \cap d_{i}$. For example, see Figure 2 where $\alpha$ and $\gamma$ are lowest but $\beta$ is not.

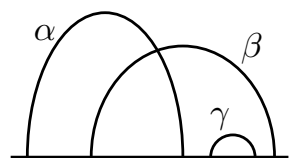

Figure 2. Lowest arcs $\alpha$ and $\gamma$

The arc $\alpha$ comes from some $D \in P$. Now cut $D$ along $\alpha$, discard the section not containing $a_{i}$ and glue in a disc parallel to $d_{i}$. This results in a new disc $D^{*}$ whose intersection with $d_{i}$ contains at least one less arc.

Any disc $E \in P$ for which $E \cap D=a_{j}$ or $\emptyset$ also has $E \cap D^{*}=a_{j}$ or $\emptyset$ respectively; if not $E$ must intersect $D^{*}$ in the section parallel to $d_{i}$ and this contradicts the condition that $\alpha$ is a lowest arc. Therefore the triple $\left(P, D, D^{*}\right)$ form a valid substitution and, by Lemma 3.2 , we can replace $D$ with $D^{*}$ to get a new homotopic loop $P^{*}$.

We now have a homotopic loop $P^{*}$ that has fewer intersections with $d_{1} \cup d_{2} \cup \ldots \cup d_{n}$. So by induction on the number of intersections we have proved the claim.

So we may assume that the path $P$ meets $d_{1}, d_{2}, \ldots, d_{n}$ only in the arcs $a_{1}, a_{2}, \ldots, a_{n}$. Therefore, for each $D \in P$ cutting out the arc $a_{i}$, the triple $\left(P, D, d_{i}\right)$ forms a valid substitution and so by in turn replacing each $D \in P$ with $d_{i}$ we see that $P$ is homotopic to the constant path $v_{0}$. The connectedness of $\overline{\mathbf{X}}_{n}$ follows by taking $P$ to be a constant loop.

Up to homotopy the group $\mathbf{H}_{2 n}$ acts on $\left(H^{3}, a_{*}\right)$ by homeomorphisms, therefore it takes cut systems to cut systems. The edges and faces of $\overline{\mathbf{X}}_{n}$ are determined by the intersections of pairs of discs, hence this action on $\overline{\mathbf{X}}_{n}^{0}$ extends to a cellular action on $\overline{\mathbf{X}}_{n}$.

Theorem 3.4. The action of $\mathbf{H}_{2 n}$ on $\overline{\mathbf{X}}_{n}^{0}$ is transitive. 
Proof. Given a vertex $\left\langle D_{1}, D_{2}, \ldots, D_{n}\right\rangle$ of $\overline{\mathbf{X}}_{n}$, if we take each $i$ in turn and look at the intersection of $D_{i}$ with $\partial H^{3}$. We see that this defines a path from one end of $a_{i}$ to the other. If we now move one end around this path until it is close to the other and then move it straight back to its starting point we have an element of $\mathbf{H}_{2 n}$ that moves $D_{i}$ to $d_{i}$. Combining all of these we see that $\left\langle D_{1}, D_{2}, \ldots, D_{n}\right\rangle$ is in the orbit of $v_{0}$, ie the action is transitive on $\overline{\mathbf{X}}_{n}^{0}$.

\section{The complex $\mathrm{X}_{n}$}

We now construct a subcomplex $\mathbf{X}_{n}$ of $\overline{\mathbf{X}}_{n}$ with the same vertex set but with fewer edges and faces.

Given an edge $e=\left(\langle D\rangle,\left\langle D^{\prime}\right\rangle\right)$ of $\overline{\mathbf{X}}_{n}$ define its length, $l(e)$, to be the number of arcs underneath $D \cup D^{\prime}$. In other words, since $H^{3} \backslash D \cup D^{\prime}$ has two components, one bounded and one unbounded, we can define the length as follows

$$
l(e)=\#\left\{i \mid a_{i} \text { is contained in the bounded component of } H^{3} \backslash D \cup D^{\prime}\right\} .
$$

Given two edges $e$ and $e^{\prime}$ with the same length there exists an element of $\mathbf{H}_{2 n}$ taking $e$ to $e^{\prime}$.

We will say that a rectangle $\left(\langle D, E\rangle,\left\langle D^{\prime}, E\right\rangle,\left\langle D^{\prime}, E^{\prime}\right\rangle,\left\langle D, E^{\prime}\right\rangle\right)$ is nested if $E \cup E^{\prime}$ lies in the bounded component of $H^{3} \backslash D \cup D^{\prime}$ or vice versa, ie if one pair of changing discs lies underneath the other.

For $i \leq j$ let $\mathcal{T}_{i j}$ denote the subcomplex consisting of all triangular faces of $\overline{\mathbf{X}}_{n}$ with shortest two edges of length $i$ and $j$. Note, this implies that the remaining edge has length $i+j$. Given a rectangular face of $\overline{\mathbf{X}}_{n}$ we have two cases depending on whether it is nested or not. Let $\mathcal{R}_{i j}$ denote the subcomplex consisting of all rectangular nested faces with inner edge of length $i$ and outer edge of length $j$. For $i \leq j$ let $\mathcal{S}_{i j}$ denote the subcomplex consisting of all non-nested rectangular faces with edges of length $i$ and $j$.

Definition 4.1. Let $\mathbf{X}_{n}$ be the subcomplex of $\overline{\mathbf{X}}_{n}$ with the same vertex set, all edges of length 1 and 2 and all faces from $\mathcal{R}_{12}, \mathcal{S}_{11}$ and $\mathcal{T}_{11}$, ie $\mathbf{X}_{n}=\mathcal{R}_{12} \cup \mathcal{S}_{11} \cup \mathcal{T}_{11}$. As the length of an edge is invariant under the action of $\mathbf{H}_{2 n}$ on $\overline{\mathbf{X}}_{n}$ this action preserves each $\mathcal{T}_{i j}, \mathcal{R}_{i j}$ and $\mathcal{S}_{i j}$ and so preserves $\mathbf{X}_{n}$.

A vertex $v=\left\langle D_{1}, \ldots, D_{n}\right\rangle$ is completely determined by the intersection of the discs $D_{i}$ with $\partial H^{3}$. Using this we can define the vertices $x_{i}$ for $0 \leq i \leq n-1, y_{i j}$ for $0 \leq i \leq n-2$ and $j=0$ or $i<j \leq n-1$ and $z_{i j}$ for $0 \leq i, j, i+j \leq n-2$ as in Figure 3. So we have $l\left(v_{0}, x_{i}\right)=i, l\left(v_{0}, y_{0 j}\right)=j, l\left(v_{0}, y_{i 0}\right)=i, l\left(v_{0}, z_{i 0}\right)=i$ and $l\left(v_{0}, z_{0 j}\right)=j$. Note, there is some redundancy in this notation, ie $x_{i}=y_{0 i}$ and $x_{0}=y_{00}=z_{00}=v_{0}$.

We now define the faces $R_{i j} \in \mathcal{R}_{i j}, S_{i j} \in \mathcal{S}_{i j}, T_{i j} \in \mathcal{T}_{i j}$ of $\overline{\mathbf{X}}_{n}$ as follows.
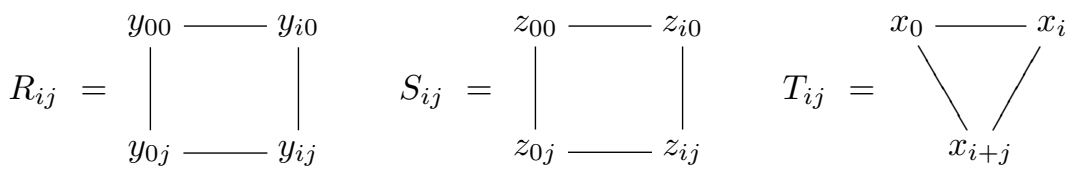

For every face in $\overline{\mathbf{X}}_{n}$ there is an element of $\mathbf{H}_{2 n}$ taking it to one of these representatives. 


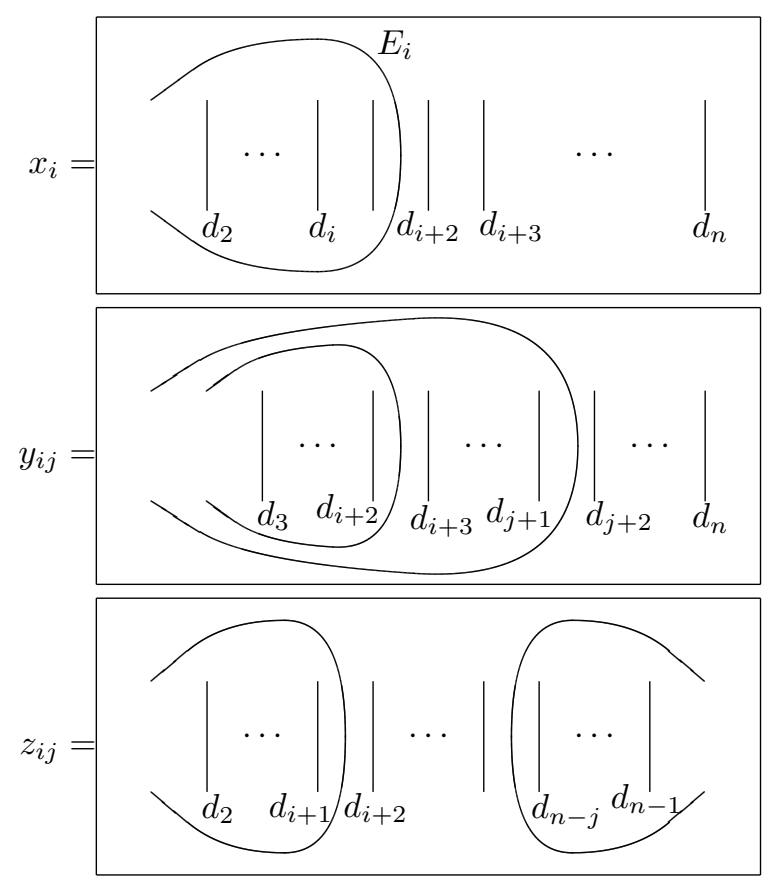

FIGURE 3 . The vertices $x_{i}, y_{i j}$ and $z_{i j}$

Theorem 4.2. The complex $\mathbf{X}_{n}$ is simply connected.

Proof. Figure 4 shows that the boundary of each of the faces $R_{i j}$ for $1<i<j$ and $S_{i j}$ for $1<i, j$ can be expressed as the boundary of a union of faces with shorter edges. The first column shows how to replace faces where the first index is not 1 . Then the second column can be used to reduce the second index to either 2 or 1 respectively.

As each of the rectangular faces can be moved to one of $R_{i j}$ or $S_{i j}$ by some element of $\mathbf{H}_{2 n}$ it follows that every loop in $\mathbf{X}_{n}$ is null-homotopic in

$$
\mathcal{R}_{12} \cup \mathcal{S}_{11} \cup \bigcup_{1 \leq i \leq j \leq n} \mathcal{T}_{i j}
$$

Let the $E_{i}$ be the discs as shown in Figure 3 , ie $x_{i}=\left\langle E_{i}, d_{2}, d_{3}, \ldots, d_{n}\right\rangle$. For $j>2$ let $A_{j}$ the be full subcomplex of $\overline{\mathbf{X}}_{n}$ containing all the vertices "between" $x_{0}$ and $x_{j}$, ie

$$
\begin{aligned}
& A_{j}^{0}=\left\{\left\langle D, d_{2}, d_{3}, \ldots, d_{n}\right\rangle \in \overline{\mathbf{X}}_{n}^{0}\right. \\
&\left.\mid D \neq d_{0} \text { or } E_{j}, \text { interior of } D \subset \text { bounded component of } H^{3} \backslash E_{0} \cup E_{j}\right\} .
\end{aligned}
$$

Choose $x_{1}$ as a base point of $A_{j}$. 

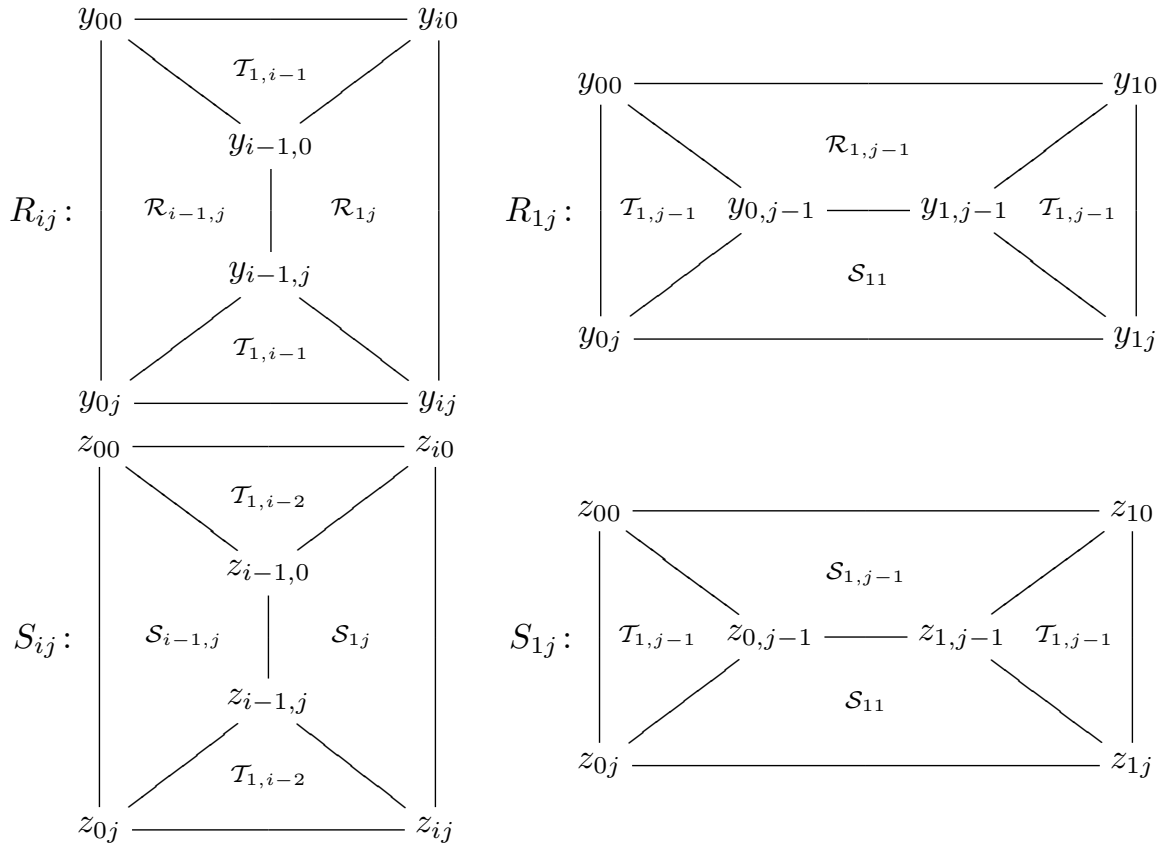

FIGURE 4. Decomposing rectangular faces

For every edge $(u, v)$ of $A_{j}$ we have the following two triangles in $\overline{\mathbf{X}}_{n}$. Note that all edges have length less than $j$.

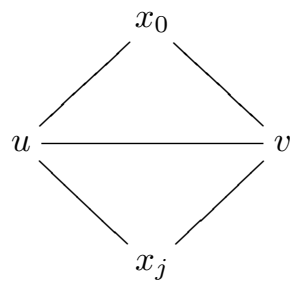

Lemma 4.3. The subcomplex $A_{j}$ is path connected.

Proof. Given a vertex $v=\left\langle D, d_{2}, \ldots d_{n}\right\rangle \in A_{j}^{0}$. First suppose that for some $2<i \leq j$ there exists a path $\gamma$ on $\partial H^{3}$ from $d_{i}$ to $E_{j}$ such that $\gamma$ does not cross $E_{1}, D$ or $d_{l}$ for $l \neq i$. Let $D^{\prime}$ be a disc parallel to $E_{j}$ except in a neighbourhood of $\gamma$ where we glue in the boundary of a neighbourhood of $\gamma \cup d_{i}$. Then there is a path $\left(v, v^{\prime}, x_{1}\right)$ in $A_{j}$ where $v^{\prime}=\left\langle D^{\prime}\right\rangle$. See Figure 5 .

Now suppose that no such path exists on $\partial H^{3}$. Each vertex $u=\left\langle D_{u}\right\rangle$ of $A_{j}$ partitions the set $\left\{d_{2}, d_{3}, \ldots d_{j+1}\right\}$ into two non-empty subsets. The first containing those discs that are between $d_{1}$ and $D_{u}$, the second those between $D_{u}$ and $E_{j}$. (If one of these sets were empty then we would have that either $D_{u}=d_{1}$ or $D_{u}=E_{j}$.) As $j>2$ at least one of these sets contains more than one disc. Choose an $i \neq 1$ such that $d_{i}$ is in this set. 


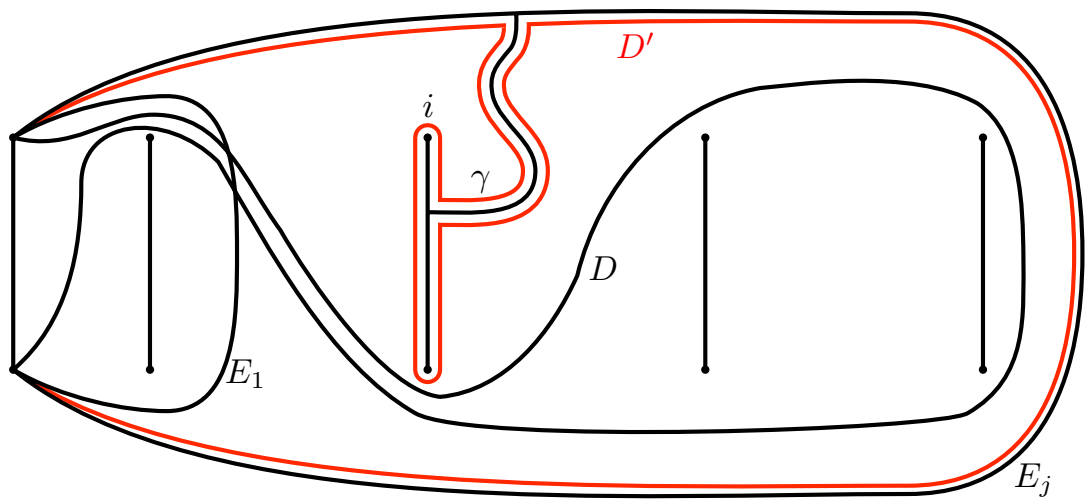

FiguRE 5. Tunnelling along $\gamma$

Now draw a path $\gamma$ on $\partial H^{3}$ from $d_{i}$ to $E_{j}$ that doesn't intersect $d_{l}$ for $l=3, \ldots, j$ or $E_{1}$ and only intersects $D$ transversely. Starting at $d_{i}$ move along $\gamma$ and label the successive points of $\gamma \cap D$ as $p_{1}, p_{2}, \ldots, p_{k}$. Now we can construct a sequence of discs $D=D^{0}, D^{1}, \ldots, D^{k}$ where each $D^{l+1}$ is parallel to $D^{l}$ except in a neighbourhood of $p_{l+1}$ where we glue in the boundary of a sufficiently small neighbourhood of the disc $d_{i}$ and the segment of $\gamma$ up to $p_{l+1}$. With each successive $D^{l}$ the disc $d_{i}$ moves from one side of the partition to the other. At each step neither side of the partition is empty so $\left\langle D^{l}\right\rangle$ is a vertex of $A_{j}$. This gives a path $\left(v=\left\langle D^{0}\right\rangle,\left\langle D^{1}\right\rangle, \ldots,\left\langle D^{k}\right\rangle\right)$ in $A_{j}$. Now, $\left\langle D^{k}\right\rangle$ satisfies the hypothesis above, therefore this path can be continued to the base point $x_{1}$.

We can now complete the proof of Theorem 4.2. So far we have shown that any loop in $\mathbf{X}_{n}$ is the boundary of a union of faces in $\mathcal{R}_{12} \cup \mathcal{S}_{11} \cup \bigcup_{1 \leq i \leq j \leq n} \mathcal{T}_{i j}$. For a given loop take an edge $(u, v)$ of maximal length $j$ in this union. If $j>2$ then the faces on either side of $(u, v)$ must be triangular with the remaining edges of length less than $j$. So we have the following situation for some $u^{\prime}, v^{\prime} \in \overline{\mathbf{X}}_{n}^{0}$.



By Lemma 4.3 we can replace these two triangles with the following.

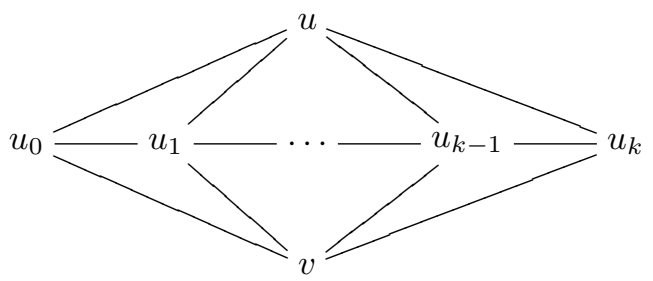


Where $u_{0}=u^{\prime}$ and $u_{k}=v^{\prime}$. Here each edge has length less than $j$. Therefore all edges of length greater that 2 can be replaced and so the loop is null-homotopic in $\mathbf{X}_{n}$.

\section{Calculating the presentation}

By Section 4 we have an $\mathbf{H}_{2 n}$-action on a simply connected cellular complex. So we can now follow the method given in Section 2.

Using the fact that $\mathbf{H}_{2 n}$ is a subgroup of $\mathbf{B}_{2 n}$, we can define the following elements of $\mathbf{H}_{2 n}$ in terms of $\sigma_{1}, \ldots, \sigma_{2 n-1}$ the generators of $\mathbf{B}_{2 n}$.

$$
\begin{array}{lll}
r_{1}=\sigma_{2} \sigma_{1} \sigma_{3}^{-1} \sigma_{2}^{-1} & & \\
r_{2}=\sigma_{4} \sigma_{3} \sigma_{2} \sigma_{1} \sigma_{5}^{-1} \sigma_{4}^{-1} \sigma_{3}^{-1} \sigma_{2}^{-1} & & \text { for } i \in\{1, \ldots, n-1\} \\
s_{i}=\sigma_{2 i} \sigma_{2 i-1} \sigma_{2 i+1} \sigma_{2 i} & & \text { for } i \in\{1, \ldots, n\} \\
t_{i}=\sigma_{2 i-1} & \text { i }
\end{array}
$$

So $r_{1}$ is the first arc passing through the second, $r_{2}$ is the first two arcs passing through the third, $s_{i}$ is the $i$ th and $i+1$ st arcs crossing and $t_{i}$ is the $i$ th arc performing a half twist. Subsequently we will prove that these generate $\mathbf{H}_{2 n}$.

Proposition 5.1. The stabiliser of the vertex $v_{0}$ is isomorphic to the framed braid group and hence has a presentation $\left\langle S_{0} \mid R_{0}\right\rangle$ where

$$
\begin{aligned}
& S_{0}=\left\{s_{1}, s_{2}, \ldots, s_{n-1}, t_{1}, t_{2}, \ldots, t_{n}\right\} \\
& R_{0}=\left\{\begin{array}{cl}
s_{i} s_{j}=s_{j} s_{i} & \text { for }|i-j|>1,
\end{array}\right. \\
& s_{i} s_{j} s_{i}=s_{j} s_{i} s_{j} \quad \text { for }|i-j|=1 \text {, } \\
& t_{i} t_{j}=t_{j} t_{i} \quad \text { for all } i, j \text {, } \\
& s_{i} t_{j}=t_{j} s_{i} \quad \text { if } j \notin\{i, i+1\} \text {, } \\
& \left.s_{i} t_{j}=t_{k} s_{i} \quad \text { if }\{i, i+1\}=\{j, k\} \quad\right\}
\end{aligned}
$$

Proof. If we restrict to $\partial H^{3}$, elements of $\mathbf{H}_{2 n}$ can be thought of as motions of the end points of the $a_{i}$. For elements of the vertex stabiliser this motion moves the $d_{i} \cap \partial H^{3}$ among themselves, ie this is the fundamental group of configurations of $n$ line segments in the plain, the framed braid group.

We have two edge orbits, one consisting of edges of length 1 and the other consisting of edges of length 2. Note that our choice of $r_{1}$ and $r_{2}$ mean that

$$
\begin{aligned}
& \left(v_{0}, v_{0} \cdot r_{1}\right) \in l^{-1}(1) \\
& \left(v_{0}, v_{0} \cdot r_{2}\right) \in l^{-1}(2) .
\end{aligned}
$$

For $i=1,2$, let $I_{i}$ denote the stabiliser of the edge $\left(v_{0}, v_{0} \cdot r_{i}\right)$, ie the subgroup of all elements that fix both $v_{0}$ and $v_{0} \cdot r_{i}$.

Proposition 5.2. The subgroups $I_{1}$ and $I_{2}$ are generated as follows.

$$
\begin{aligned}
& I_{1}=\left\langle t_{2}, t_{3}, \ldots, t_{n}, s_{3}, s_{4}, \ldots, s_{n-1}, s_{1} s_{1} t_{1} t_{1}, s_{2} s_{1} s_{1} s_{2}\right\rangle \\
& I_{2}=\left\langle t_{2}, t_{3}, \ldots, t_{n}, s_{2}, s_{4}, s_{5}, \ldots, s_{n-1}, s_{1} s_{2} s_{2} s_{1} t_{1} t_{1}, s_{3} s_{2} s_{1} s_{1} s_{2} s_{3}\right\rangle
\end{aligned}
$$


Proof. For $I_{1}\left[I_{2}\right]$ the motion of the $d_{i}$ outside of $d_{1} \cup E_{2}\left[d_{1} \cup E_{3}\right]$ is generated by $t_{3}, t_{4}, \ldots, t_{n}, s_{3}, s_{4}, \ldots, s_{n-1} \quad$ and $s_{2} s_{1} s_{1} s_{2} \quad\left[t_{4}, t_{5}, \ldots, t_{n}, \quad s_{4}, s_{5}, \ldots, s_{n-1} \quad\right.$ and $\left.s_{3} s_{2} s_{1} s_{1} s_{2} s_{3}\right]$, the motion of the $d_{i}$ inside $d_{1} \cup E_{2}\left[d_{1} \cup E_{3}\right]$ is generated by $t_{2}\left[t_{2}, t_{3}, s_{2}\right]$ and the motion of $d_{1} \cup E_{2}\left[d_{1} \cup E_{3}\right]$ is generated by $s_{1} s_{1} t_{1} t_{1}\left[s_{1} s_{2} s_{2} s_{1} t_{1} t_{1}\right]$.

We are now ready to calculate relations for $R_{1}, R_{2}$ and $R_{3}$. The following relations are easily verifiable, in fact most of them take place in $\mathbf{B}_{8}$.

The $R_{1}$ relations. To calculate the $R_{1}$ relations we have to find, for each edge orbit representative $\left(v_{0}, v_{0} \cdot r_{i}\right)$ and each generator $t$ of $I_{i}$, a word $h$ in $S_{0}$ such that $r_{i} t r_{i}^{-1}=h$. One possibility is the following.
$\left(R_{1} 1\right)$

$$
\begin{aligned}
r_{1} t_{2} r_{1}^{-1} & =t_{1} & & \\
r_{1} t_{k} r_{1}^{-1} & =t_{k} & & \text { for } k>2 \\
r_{1} s_{k} r_{1}^{-1} & =s_{k} & & \text { for } k>2 \\
r_{1} s_{1} s_{1} t_{1} t_{1} r_{1}^{-1} & =s_{1} s_{1} t_{2} t_{2} & & \\
r_{1} s_{2} s_{1} s_{1} s_{2} r_{1}^{-1} & =s_{2} s_{1} s_{1} s_{2} & &
\end{aligned}
$$

The $R_{2}$ relations. To calculate the $R_{2}$ relations we need to find, for each edge orbit representative $\left(v_{0}, v_{0} \cdot r_{i}\right)$, an h-product $r_{i} h r_{i}$ for the path $\left(v_{0}, v_{0} \cdot r_{i}, v_{0}\right)$ and a word $h^{\prime}$ in $S_{0}$ such that $r_{i} h r_{i}=h^{\prime}$.

$$
\begin{aligned}
r_{1} t_{1} s_{1} r_{1} & =s_{1} t_{1} \\
r_{2} s_{1} t_{2} s_{2} & r_{2} \\
& =s_{2} s_{1} t_{1}
\end{aligned}
$$

The $R_{3}$ relations. To calculate the $R_{3}$ relations we need to find, for each edge orbit, an h-product representing the boundary of a face in the orbit and an equivalent word in $S_{0}$. The following are such relations for the $\mathcal{S}_{11}, \mathcal{R}_{12}$ and $\mathcal{T}_{11}$ orbits respectively.

$$
\begin{gathered}
r_{1} s_{1} s_{2} s_{3} s_{1} s_{2} r_{1} s_{1} s_{2} s_{3} s_{1} s_{2} t_{2} t_{4} r_{1} s_{2} s_{3} s_{1} s_{2} r_{1} \\
=s_{1} s_{2} s_{3} s_{1} s_{2} s_{1} s_{2} s_{1} s_{3} s_{2} s_{2} s_{3} s_{1} s_{2} t_{1} t_{3} \\
r_{1} r_{2} s_{1} s_{2} s_{1} t_{2} t_{3} r_{1} r_{2}=s_{2} s_{1} s_{2} t_{1} t_{2} \\
r_{2} s_{1} t_{2} r_{1} s_{2} s_{1} r_{1}=s_{1} s_{2} s_{1} t_{1}
\end{gathered}
$$

If we use a different set of generators, similar to those found by Hilden, then we can get a more braid like presentation. Let $p_{i}=\sigma_{2 i} \sigma_{2 i-1} \sigma_{2 i+1}^{-1} \sigma_{2 i}^{-1}$ for $1 \leq i<n$. So $p_{i}$ is the $i$ th arc passing under the $i+1$ st arc, see Figure 7 . 


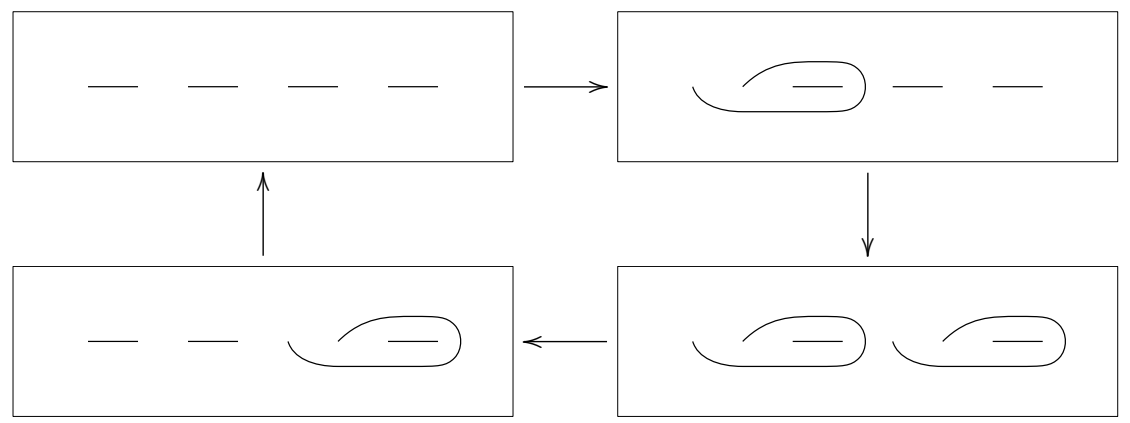

Figure 6 . The path given by the h-product on the LHS of $\left(R_{3} 1\right)$

Theorem 5.3. The group $\mathbf{H}_{2 n}$ has a presentation with generators $p_{i}, s_{j}$ and $t_{k}$ for $1 \leq i, j<n$ and $1 \leq k \leq n$ and the following relations.

$$
\begin{aligned}
p_{i} p_{j} & =p_{j} p_{i} \\
p_{i} p_{j} p_{i} & =p_{j} p_{i} p_{j} \\
s_{i} s_{j} & =s_{j} s_{i} \\
s_{i} s_{j} s_{i} & =s_{j} s_{i} s_{j} \\
p_{i} s_{j} & =s_{j} p_{i} \\
p_{i} s_{i+1} s_{i} & =s_{i+1} s_{i} p_{i+1} \\
p_{i+1} p_{i} s_{i+1} & =s_{i} p_{i+1} p_{i} \\
p_{i+1} s_{i} s_{i+1} & =s_{i} s_{i+1} p_{i} \\
p_{i} t_{i} s_{i} p_{i} & =s_{i} t_{i} \\
p_{i} t_{j} & =t_{j} p_{i} \\
p_{i} t_{i+1} & =t_{i} p_{i} \\
s_{i} t_{j} & =t_{j} s_{i} \\
s_{i} t_{j} & =t_{k} s_{i} \\
t_{i} t_{j} & =t_{j} t_{i}
\end{aligned}
$$$$
\text { for }|i-j|>1
$$$$
\text { for }|i-j|=1
$$$$
\text { for }|i-j|>1
$$$$
\text { for }|i-j|=1
$$$$
\text { for }|i-j|>1
$$$$
\text { for } j \neq i \text {, or } i+1
$$$$
\text { if } j \neq i \text { or } i+1
$$$$
\text { if }\{i, i+1\}=\{j, k\}
$$$$
\text { for } 1 \leq i, j \leq n
$$<smiles>CCC(CC)CC(CC)CC(CC)CC</smiles><smiles>CCC(CC)CC(CC(C)C)CC(CC)CC</smiles>

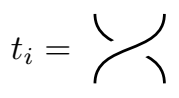

FiguRE 7. Generators of $\mathbf{H}_{2 n}$

These generators and relations can be represented pictorially as in Figure 8 and Figure 9. 


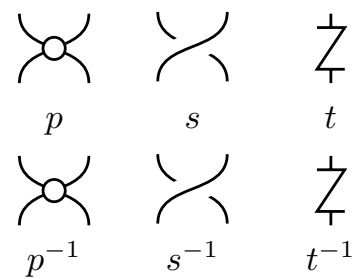

Figure 8. Pictorial representation of the $p, s, t$ and their inverses

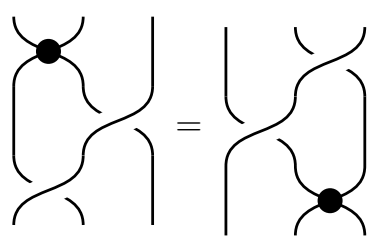

(P6)

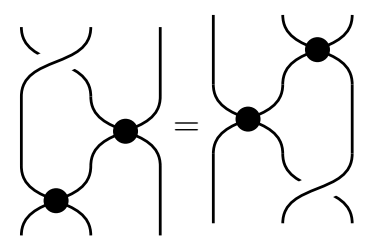

(P7)



(P9)

Figure 9. Pictorial representation of (P6), (P7) and (P9)

Proof. Since $\mathbf{H}_{2 n}$ is a subgroup of the braid group it is easy to check that these relations all hold. So it remains to prove that each of the relations in $R_{0}, R_{1}, R_{2}$ and $R_{3}$ can be deduced from (P1)-(P14) using the fact that $r_{1}=p_{1}$ and $r_{2}=p_{2} p_{1}$. First note that $R_{0}$ is a subset of these relations. The relations $\left(R_{1} 1\right),\left(R_{1} 2\right),\left(R_{1} 3\right)$ and $\left(R_{2} 1\right)$ follow directly from (P11), (P10), (P5) and (P9) respectively. The remaining relations can be deduced as follows. Some of these relations are quite long and are perhaps better understood using pictorial representations. For the longest, $\left(R_{3} 1\right)$, see Figure 10 for a pictorial version.

$$
\begin{aligned}
& \left(R_{1} 4\right): \quad r_{1} s_{1} s_{1} t_{1} t_{1} r_{1}^{-1}=p_{1} s_{1} s_{1} t_{1} t_{1} p_{1}^{-1} \\
& =p_{1} t_{1} s_{1} s_{1} t_{1} p_{1}^{-1} \\
& =s_{1} t_{1} p_{1}^{-1} s_{1} t_{1} p_{1}^{-1} \\
& \begin{array}{l}
=s_{1} t_{1} t_{1} s_{1} \\
=s_{1} \\
s_{1} t_{2} t_{2}
\end{array} \\
& \left(R_{1} 5\right): \quad r_{1} s_{2} s_{1} s_{1} s_{2} r_{1}^{-1}=\underline{p_{1} s_{2} s_{1}} s_{1} s_{2} p_{1}^{-1} \\
& =s_{2} s_{1} p_{2} s_{1} s_{2} p_{1}^{-1} \\
& =s_{2} s_{1} s_{1} s_{2} \\
& \left(R_{1} 6\right): \quad \quad r_{2} t_{2} r_{2}^{-1}=p_{2} p_{1} t_{2} p_{1}^{-1} p_{2}^{-1} \\
& =\underline{p_{2} t_{1}} p_{2}^{-1} \\
& =t_{1} \\
& \left(R_{1} 7\right): \quad r_{2} t_{3} r_{2}^{-1}=p_{2} p_{1} t_{3} p_{1}^{-1} p_{2}^{-1} \\
& =\underline{p_{2}} \overline{t_{3} p_{2}^{-1}} \\
& =t_{2} \\
& \left(R_{1} 8\right): \quad \quad r_{2} t_{k} r_{2}^{-1}=p_{2} p_{1} t_{k} p_{1}^{-1} p_{2}^{-1}
\end{aligned}
$$




$$
\begin{aligned}
& =\underline{p_{2} t_{k}} p_{2}^{-1} \\
& =\bar{t}_{k} \\
& \begin{aligned}
\left(R_{1} 9\right): & r_{2} s_{2} r_{2}^{-1} \\
& =\underline{p_{2} p_{1} s_{2} p_{1}^{-1} p_{2}^{-1}} \\
& =s_{1}
\end{aligned} \\
& \begin{aligned}
\left(R_{1} 10\right): \quad & r_{2} s_{k} r_{2}^{-1} \\
& =\underline{p_{2} p_{1} s_{k}} p_{1}^{-1} p_{2}^{-1} \\
& =s_{k}
\end{aligned}
\end{aligned}
$$

To deduce $\left(R_{1} 11\right)$ we make use of the following deduction.

$(\star)$

$$
\begin{aligned}
& \underline{p_{2} p_{1}} s_{1} s_{2} t_{3} p_{2} p_{1} \\
& =s_{1}^{-1} p_{2} p_{1} s_{2} s_{1} s_{2} t_{3} p_{2} p_{1} \\
& =s_{1}^{-1} p_{2} p_{1} \underline{s_{1} s_{2} s_{1}} t_{3} p_{2} p_{1} \\
& =s_{1}^{-1} p_{2} p_{1} \bar{t}_{1} s_{1} s_{2} s_{1} p_{2} p_{1} \\
& =s_{1}^{-1} p_{2} p_{1} t_{1} s_{1} \bar{p}_{1} s_{2} s_{1} p_{1} \\
& =s_{1}^{-1} p_{2} s_{1} t_{1} s_{2} s_{1} p_{1} \\
& =s_{2} p_{1} s_{2}^{-1} \underline{t_{1} s_{2}} s_{1} p_{1} \\
& =s_{2} p_{1} t_{1} s_{1} p_{1} \\
& =s_{2} s_{1} t_{1}
\end{aligned}
$$

$$
\begin{aligned}
& \left(R_{1} 11\right): \quad r_{2} s_{1} s_{2} s_{2} s_{1} t_{1} t_{1} r_{2}^{-1}=p_{2} p_{1} s_{1} s_{2} s_{2} s_{1} t_{1} t_{1} p_{1}^{-1} p_{2}^{-1} \\
& =p_{2} p_{1} s_{1} s_{2} \overline{t_{3} s_{2} s_{1}} t_{1} p_{1}^{-1} p_{2}^{-1} \\
& =p_{2} p_{1} s_{1} s_{2} t_{3} \overline{p_{2} p_{1}} s_{1} s_{2} t_{3} \\
& =s_{2} s_{1} t_{1} s_{1} s_{2} t_{3} \\
& =s_{2} s_{1} s_{1} s_{2} t_{3} t_{3} \\
& \left(R_{1} 12\right): \quad r_{2} s_{3} s_{2} s_{1} s_{1} s_{2} s_{3} r_{2}^{-1}=p_{2} \underline{p_{1} s_{3}} s_{2} s_{1} s_{1} s_{2} s_{3} p_{1}^{-1} p_{2}^{-1} \\
& =p_{2} s_{3} \underline{p_{1}} s_{2} s_{1} s_{1} s_{2} s_{3} p_{1}^{-1} p_{2}^{-1} \\
& =p_{2} s_{3} s_{2} s_{1} \underline{p_{2}} s_{1} s_{2} s_{3} p_{1}^{-1} p_{2}^{-1} \\
& =p_{2} s_{3} s_{2} s_{1} s_{1} s_{2} \underline{p_{1}} s_{3} p_{1}^{-1} p_{2}^{-1} \\
& =\underline{p_{2} s_{3} s_{2}} s_{1} s_{1} s_{2} s_{3} p_{2}^{-1} \\
& =s_{3} s_{2} p_{3} s_{1} s_{1} s_{2} s_{3} p_{2}^{-1} \\
& =s_{3} s_{2} s_{1} s_{1} p_{3} s_{2} s_{3} p_{2}^{-1} \\
& =s_{3} s_{2} s_{1} s_{1} s_{2} s_{3} \\
& \left(R_{2} 2\right): \quad r_{2} s_{1} t_{2} s_{2} r_{2}=p_{2} p_{1} s_{1} \underline{t_{2} s_{2} p_{2}} p_{1} \\
& =p_{2} p_{1} s_{1} p_{2}^{-1} s_{2} t_{2} p_{1} \\
& =p_{2} p_{1} s_{1} p_{2}^{-1} s_{2} t_{2} s_{1}^{-1} t_{1}^{-1} p_{1}^{-1} s_{1} t_{1} \\
& =p_{2} p_{1} s_{1} p_{2}^{-1} s_{2} \overline{s_{1}^{-1} p_{1}^{-1}} s_{1} t_{1} \\
& =p_{2} p_{1} \bar{s}_{2}^{-1} p_{1}^{-1} s_{2} s_{1} s_{2} s_{1}^{-1} p_{1}^{-1} s_{1} t_{1} \\
& =p_{2} p_{1} s_{2}^{-1} p_{1}^{-1} s_{1} s_{2} p_{1}^{-1} s_{1} t_{1} \\
& =p_{2} p_{1} s_{2}^{-1} p_{1}^{-1} \overline{p_{2}^{-1} s_{1} s_{2}} s_{1} t_{1} \\
& =s_{2} s_{1} t_{1}
\end{aligned}
$$

$(\mathrm{P} 13)^{2}$

$\left(R_{3} 1\right): \quad r_{1} s_{1} s_{2} s_{3} s_{1} s_{2} r_{1} s_{1} s_{2} s_{3} s_{1} s_{2} t_{2} t_{4} r_{1} s_{2} s_{3} s_{1} s_{2} r_{1}$

$$
\begin{aligned}
& =p_{1} s_{1} s_{2} s_{3} s_{1} s_{2} p_{1} s_{1} s_{2} s_{3} \underline{s_{1} s_{2} t_{2}} t_{4} p_{1} s_{2} s_{3} s_{1} s_{2} p_{1} \\
& =p_{1} s_{1} s_{2} s_{3} s_{1} s_{2} p_{1} \underline{s_{1} s_{2} s_{3} t_{3} s_{1} s_{2}} t_{4} p_{1} s_{2} s_{3} s_{1} s_{2} p_{1}
\end{aligned}
$$

$(\mathrm{P} 13)(\mathrm{P} 12)^{2}$ 
$=p_{1} s_{1} s_{2} s_{3} s_{1} s_{2} p_{1} t_{4} s_{1} s_{2} s_{3} s_{1} s_{2} t_{4} p_{1} s_{2} s_{3} s_{1} s_{2} p_{1}$

$=p_{1} s_{1} s_{2} s_{3} t_{4} s_{1} s_{2} p_{1} s_{1} s_{2} s_{3} s_{1} s_{2} t_{4} p_{1} s_{2} s_{3} s_{1} s_{2} p_{1}$

$=p_{1} \overline{t_{1} s_{1} s_{2} s_{3}} s_{1} s_{2} p_{1} s_{1} s_{2} s_{3} s_{1} s_{2} t_{4} p_{1} s_{2} s_{3} s_{1} s_{2} p_{1}$

$=p_{1} t_{1} s_{1} s_{2} s_{3} s_{1} s_{2} p_{1} s_{1} s_{2} s_{3} s_{1} s_{2} p_{1} t_{4} s_{2} s_{3} s_{1} s_{2} p_{1}$

$=p_{1} t_{1} s_{1} s_{2} s_{3} s_{1} s_{2} p_{1} s_{1} \overline{p_{3} s_{2} s_{3} s_{1} s_{2}} t_{4} s_{2} s_{3} s_{1} s_{2} p_{1}$

$=p_{1} t_{1} s_{1} s_{2} s_{3} s_{1} s_{2} p_{3} p_{1} s_{1} s_{2} s_{3} s_{1} s_{2} t_{4} s_{2} s_{3} s_{1} s_{2} p_{1}$

$=p_{1} t_{1} s_{1} s_{2} s_{1} s_{3} s_{2} p_{3} p_{1} s_{1} s_{2} s_{3} s_{1} s_{2} t_{4} s_{2} s_{3} s_{1} s_{2} p_{1}$

$=p_{1} t_{1} s_{1} s_{2} s_{1} \overline{p_{2} s_{3} s_{2}} p_{1} s_{1} s_{2} s_{3} s_{1} s_{2} t_{4} s_{2} s_{3} s_{1} s_{2} p_{1}$

$=p_{1} t_{1} s_{1} \overline{p_{1} s_{2} s_{1}} s_{3} s_{2} p_{1} s_{1} s_{2} s_{3} s_{1} s_{2} t_{4} s_{2} s_{3} s_{1} s_{2} p_{1}$

$=p_{1} t_{1} s_{1} p_{1} s_{2} s_{3} s_{1} s_{2} p_{1} s_{1} s_{2} s_{3} s_{1} s_{2} t_{4} s_{2} s_{3} s_{1} s_{2} p_{1}$

$=s_{1} t_{1} s_{2} s_{3} s_{1} s_{2} p_{1} s_{1} s_{2} s_{3} s_{1} s_{2} t_{4} s_{2} s_{3} s_{1} s_{2} p_{1}$

$=s_{1} t_{1} s_{2} s_{3} s_{1} s_{2} p_{1} s_{1} s_{2} s_{3} s_{1} s_{2} t_{4} p_{3} s_{2} s_{3} s_{1} s_{2}$

$=s_{1} t_{1} s_{2} s_{3} s_{1} s_{2} p_{1} t_{1} s_{1} s_{2} s_{3} s_{1} s_{2} p_{3} s_{2} s_{3} s_{1} s_{2}$

$=s_{1} t_{1} s_{2} s_{3} s_{1} s_{2} p_{1} t_{1} s_{1} s_{2} s_{1} s_{3} s_{2} p_{3} s_{2} s_{3} s_{1} s_{2}$

$=s_{1} t_{1} s_{2} s_{3} s_{1} s_{2} p_{1} t_{1} s_{1} \overline{p_{1} s_{2} s_{1} s_{3} s_{2}} s_{2} s_{3} s_{1} s_{2}$

$=s_{1} t_{1} s_{2} s_{3} s_{1} s_{2} s_{1} \underline{t_{1} s_{2} s_{1}} s_{3} s_{2} s_{2} s_{3} s_{1} s_{2}$

$=s_{1} t_{1} s_{2} s_{3} s_{1} s_{2} s_{1} s_{2} s_{1} t_{2} s_{3} s_{2} s_{2} s_{3} s_{1} s_{2}$

$=s_{1} t_{1} s_{2} s_{3} s_{1} s_{2} s_{1} s_{2} s_{1} s_{3} s_{2} s_{2} t_{2} s_{3} s_{1} s_{2}$

$=s_{1} t_{1} s_{2} s_{3} s_{1} s_{2} s_{1} s_{2} s_{1} s_{3} s_{2} s_{2} s_{3} s_{1} \underline{t_{1}} s_{2}$

$=s_{1} t_{1} s_{2} s_{3} s_{1} s_{2} s_{1} s_{2} s_{1} s_{3} s_{2} s_{2} s_{3} s_{1} s_{2} t_{1}$

$=s_{1} s_{2} s_{3} s_{1} s_{2} t_{3} s_{1} s_{2} s_{1} s_{3} s_{2} s_{2} s_{3} s_{1} s_{2} t_{1}$

$=s_{1} s_{2} s_{3} s_{1} s_{2} s_{1} s_{2} s_{1} t_{1} s_{3} s_{2} s_{2} s_{3} s_{1} s_{2} t_{1}$

$=s_{1} s_{2} s_{3} s_{1} s_{2} s_{1} s_{2} s_{1} s_{3} s_{2} s_{2} s_{3} s_{1} s_{2} t_{3} t_{1}$

$=s_{1} s_{2} s_{3} s_{1} s_{2} s_{1} s_{2} s_{1} s_{3} s_{2} s_{2} s_{3} s_{1} s_{2} \overline{t_{1} t_{3}}$

$$
\begin{aligned}
& \left(R_{3} 2\right): \quad r_{1} r_{2} s_{1} s_{2} s_{1} t_{3} t_{2} r_{1} r_{2}=p_{1} p_{2} p_{1} s_{1} s_{2} s_{1} t_{3} t_{2} p_{1} p_{2} p_{1} \\
& =p_{1} p_{2} p_{1} t_{1} s_{1} s_{2} s_{1} t_{2} p_{1} p_{2} p_{1} \\
& =p_{1} p_{2} s_{1} t_{1} p_{1}^{-1} s_{2} s_{1} t_{2} p_{1} p_{2} p_{1} \\
& =p_{1} p_{2} s_{1} t_{1} s_{2} s_{1} p_{2}^{-1} t_{2} \underline{p_{1} p_{2} p_{1}} \\
& =p_{1} p_{2} s_{1} t_{1} s_{2} s_{1} p_{2}^{-1} t_{2} p_{2} p_{1} p_{2} \\
& =p_{1} p_{2} s_{1} t_{1} s_{2} s_{1} t_{3} p_{1} p_{2} \\
& =p_{1} p_{2} s_{1} s_{2} t_{1} s_{1} p_{1} t_{3} p_{2} \\
& =p_{1} p_{2} s_{1} s_{2} \bar{p}_{1}^{-1} s_{1} t_{1} t_{3} p_{2} \\
& =p_{1} s_{1} \overline{s_{2} s_{1} t_{1} t_{3}} p_{2} \\
& =p_{1} s_{2} s_{1} s_{2} t_{1} t_{3} p_{2} \\
& =s_{2} s_{1} p_{2} s_{2} \underline{t_{1} t_{3} p_{2}} \\
& =s_{2} s_{1} p_{2} s_{2} t_{3} p_{2} t_{1} \\
& =s_{2} s_{1} s_{2} t_{2} t_{1} \\
& \left(R_{3} 3\right): \quad r_{2} s_{1} t_{2} r_{1} s_{2} s_{1} r_{1}=p_{2} \underline{p_{1} s_{1} t_{2} p_{1}} s_{2} s_{1} p_{1} \\
& =p_{2} s_{1} \underline{t_{1} s_{2}} s_{1} p_{1} \\
& =p_{2} s_{1} s_{2} t_{1} s_{1} p_{1} \\
& =s_{1} s_{2} \underline{p_{1} t_{1} s_{1} p_{1}} \\
& =s_{1} s_{2} s_{1} t_{1}
\end{aligned}
$$

$(\mathrm{P} 10)(\mathrm{P} 12)^{2}$

$(\mathrm{P} 13)^{3}$

(P10)

$(\mathrm{P} 8)^{2}$

$(\mathrm{P} 5)(\mathrm{P} 1)$

(P9)

$(\mathrm{P} 8)^{2}$

$(\mathrm{P} 12)^{2}(\mathrm{P} 13)^{3}$

$(\mathrm{P} 6)^{2}$

(P9)

$(\mathrm{P} 12)(\mathrm{P} 13)$

$(\mathrm{P} 12)(\mathrm{P} 13)^{2}$

$(\mathrm{P} 12)(\mathrm{P} 13)$

(P12)

$(\mathrm{P} 12)^{2}(\mathrm{P} 13)^{2}$

$(\mathrm{P} 12)(\mathrm{P} 13)^{2}$

$(\mathrm{P} 12)^{4}(\mathrm{P} 13)^{2}$

(P14)

$(\mathrm{P} 12)(\mathrm{P} 13)^{2}$

(P11)

(P12)(P10)

(P14)(P10) 


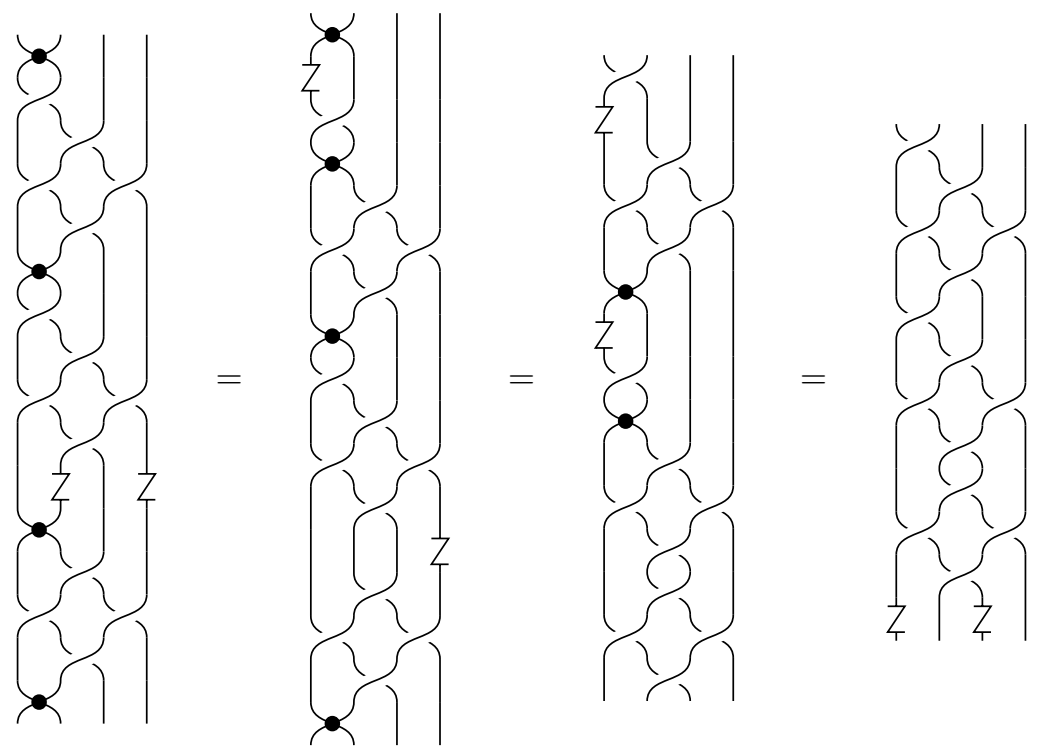

Figure 10. Deducing the $\left(R_{3} 1\right)$ relation

\section{References}

[1] J. S. Birman, On the stable equivalence of plat representations of knots and links, Canad. J. Math. 28 (1976), no. 2, 264-290.

[2] T. Brendle and A. Hatcher, Configuration spaces of rings and wickets, arXiv:0805.4354.

[3] K. S. Brown, Presentations for groups acting on simply-connected complexes, J. Pure Appl. Algebra 32 (1984), no. 1, 1-10.

[4] A. Hatcher and W. Thurston, A presentation for the mapping class group of a closed orientable surface, Topology 19 (1980), no. 3, 221-237.

[5] H. M. Hilden, Generators for two groups related to the braid group, Pacific J. Math. 59 (1975), no. $2,475-486$.

[6] F. Laudenbach, Travaux de Thurston sur les surfaces, Vol. 66 of Astérisque, chapter Présentation du groupe de difféotopies d'une surface compacte orientable, 267-282, Société Mathématique de France, Paris (1979).

[7] B. Wajnryb, A simple presentation for the mapping class group of an orientable surface, Israel J. Math. 45 (1983), no. 2-3, 157-174.

[8] — Mapping class group of a handlebody, Fund. Math. 158 (1998), no. 3, 195-228.

[9] — An elementary approach to the mapping class group of a surface, Geom. Topol. 3 (1999) 405-466 (electronic).

Mathematics Institute, University of Warwick, Coventry CV4 7AL UK

E-mail address: stephen@tawn.co.uk 